\title{
Safety of Balloon Kyphoplasty in the Treatment of Thoracic Osteoporotic Vertebral Compression Fractures in Vietnamese Patients
}

\author{
Dinh-Hoa Nguyen, $\mathrm{PhD}^{*,+, \neq}$, Duc-Dat Vu, MD ${ }^{*, \neq}$, Thi-Ngoc-Ha Doan, $\mathrm{MD}^{\S}$, Hoang-Long Vo, $\mathrm{MD}^{\S}$ \\ *Institute of Orthopedic Trauma, Viet Duc Hospital, Hanoi, \\ ${ }^{\dagger}$ Social Affair Department, Viet Duc Hospital, Hanoi, \\ ${ }^{\ddagger}$ Department of Surgery, Hai Duong Medical Technical University, Hai Duong, \\ ${ }^{\S}$ Institute for Preventive Medicine and Public Health, Hanoi Medical University, Hanoi, Vietnam
}

Background: The evidence for the efficacy and safety of balloon kyphoplasty (BKP) in treating the Vietnamese patients is sparse. There is no convincing evidence regarding BKP's efficacy in Vietnamese patients, especially in the patients with thoracic osteoporotic vertebral compression fractures (VCFs). This article aims to evaluate the outcomes of restoring the body height of the compressed thoracic vertebrae in patients undergoing BKP.

Methods: We prospectively enrolled 65 consecutive patients with thoracic VCFs (73 vertebrae) due to osteoporosis who were treated with BKP between June 2018 and May 2019.

Results: A trocar was inserted through the pedicle in $84.9 \%(62 / 73)$ and beside the pedicle in $15.1 \%(11 / 73)$. The mean amount of mixed cement injected was $4.1 \pm 1.1 \mathrm{~mL}$ (range, $1.5-7 \mathrm{~mL}$ ). Cement leakage was radiographically confirmed in $30.8 \%$ of 65 patients. Among patients with complications caused by cement extravasation, the leakage was through the anterior margin of the vertebrae in $15.4 \%$, through the vertebral disc in $12.3 \%$, and through the posterior margin of the vertebrae in $3.1 \%$. In the last $3.1 \%$ of patients, there was no clinically notable lesions of the nerve roots or spinal cord. The mean visual analog scale score decreased significantly from $7.3 \pm 1.1$ preoperatively to $3.3 \pm 0.6$ at 24 hours after surgery, and then to $1.2 \pm 1.1$ at 3 months after surgery $(p<$ 0.01). The mean reduction in Cobb angle measured on standing radiographs after treatment was $3.7^{\circ}$, showing statistical significance $(p<0.01)$.

Conclusions: BKP is a minimally invasive treatment effective for immediate pain relief, early motor rehabilitation, and humpback correction. The present study provided convincing evidence to support the use of BKP by spine surgeons and clinical specialists in treating osteoporotic thoracic VCFs in Vietnamese patients.

Keywords: Balloon kyphoplasty, Osteoporosis, Thoracic compression fracture, Vietnam

Osteoporosis is known as a condition where the density and quality of bone decrease, leading to an increased risk of fracture, especially at the hip, spine, and wrist. ${ }^{1)}$ Verte-

Received November 13, 2019; Accepted December 16, 2019

Correspondence to: Hoang-Long Vo, MD

Institute for Preventive Medicine and Public Health, Hanoi Medical University,

Hanoi 100000, Vietnam

Tel: +84-985406430, Fax: +84-438525115

E-mail: drvohoanglonghmu@daihocyhanoi.edu.vn bral compression fractures (VCFs) are a common complication in patients with osteoporosis. ${ }^{2)}$ Vertebral fractures caused by osteoporosis are usually not fatal; however, they cause serious injuries and greatly affect the economy and quality of life of patients. ${ }^{2}$ Osteoporotic VCF, the most common type of fragility fracture, was reported to affect $20 \%$ of over 50 -year-old people, in whom $45 \%$ are women. In particular, patients with untreated osteoporotic VCF may have experienced impaired quality of life. ${ }^{3)}$

Percutaneous vertebroplasty and balloon kypho-

Copyright (C) 2020 by The Korean Orthopaedic Association 
Nguyen et al. Balloon Kyphoplasty in the Treatment of Osteoporotic Vertebral Compression Fractures

Clinics in Orthopedic Surgery • Vol. 12, No. 2, $2020 \bullet$ www.ecios.org

plasty (BKP) - minimally invasive techniques-are the most popular treatment options known for safety and immediate pain relief. ${ }^{4,5)}$ The technique of vertebroplasty was first introduced by Galibert et al. ${ }^{6}$ However, vertebroplasty does not restore the height of the compressed vertebral body; therefore, a VCF may recur in the later stage of treatment and the patient may still suffer from kyphosis and chronic low back pain. Kyphoplasty, a variation of vertebroplasty, attempts to restore the height and angle of kyphotic, fractured vertebral body and to stabilize it by bone cement injection. BKP, developed in the 1990s by Dr. Mark Reiley, is the most commonly used kyphoplasty technique. ${ }^{7,8)}$ BKP involves the use of an inflatable bone tamp inserted into the collapsed vertebral body. Once inflated, the balloon elevates the end plates and thereby restores the height of the vertebral body. ${ }^{9}$

In Vietnam, in 1999, with the help of Prof. H. Deramond, the Radiology Department of Bach Mai Hospital became the first unit to successfully apply the procedure of vertebral augmentation. For many years, BKP has been considered as a regular treatment choice by Vietnamese physicians because of its safety and effectiveness. In Vietnam as well as in many countries globally, the research on vertebral augmentation is mostly focused on vertebral augmentation with bone cement injection in general VCF patients. To date, there is no convincing evidence regarding BKP's efficacy in Vietnamese patients, especially in those with thoracic osteoporotic VCF. The clinical benefits and advantages of BKP performed by Vietnamese physicians must be documented. Therefore, we aimed to evaluate the outcome of restoring the height of the collapsed thoracic vertebrae in those who underwent BKP.

\section{METHODS}

\section{Patients}

Between June 2018 and May 2019, we prospectively enrolled patients with osteoporotic thoracic VCFs who were treated with BKP. The inclusion criteria for the present study were patients who were diagnosed with VCFs due to osteoporosis (bone density with a T-score of $<2.5$ ) between thoracic vertebral levels T1 and T12 (spine X-ray), who had vertebral stem edema on magnetic resonance imaging ([MRI], low signal on T1WI images and high signal on T2WI), and who were neurologically intact. Exclusion criteria were patients with coagulation disorders, respiratory failure, sepsis, discitis, or osteomyelitis. A total of 65 patients were included in this study.

\section{Surgical Procedure}

Trocar insertion into the vertebra

All vertebroplasty practitioners (DHN, DDV) who were involved in the surgery were highly experienced with more than 10 years of experience. The patients were positioned on their stomach with their chest and sides supported by pillows. We disinfected the surgical site with betadine. A compressed vertebra was confirmed by fluoroscopy, which was used to guide a trocar into the proper position. A trocar was inserted through the vertebral pedicle for large and easily observed pedicles under C-arm and beside the pedicle for small pedicles or its anatomical abnormalities (Fig. 1). A local anesthesia was injected with lidocaine $1 \%-2 \%$ from the entry point on the external skin to the pedicle. A 0.5- $\mathrm{cm}$ skin incision was made to correspond to the point of the external skin. A needle was inserted through the incision to reach the pedicle and then replaced with the tro-
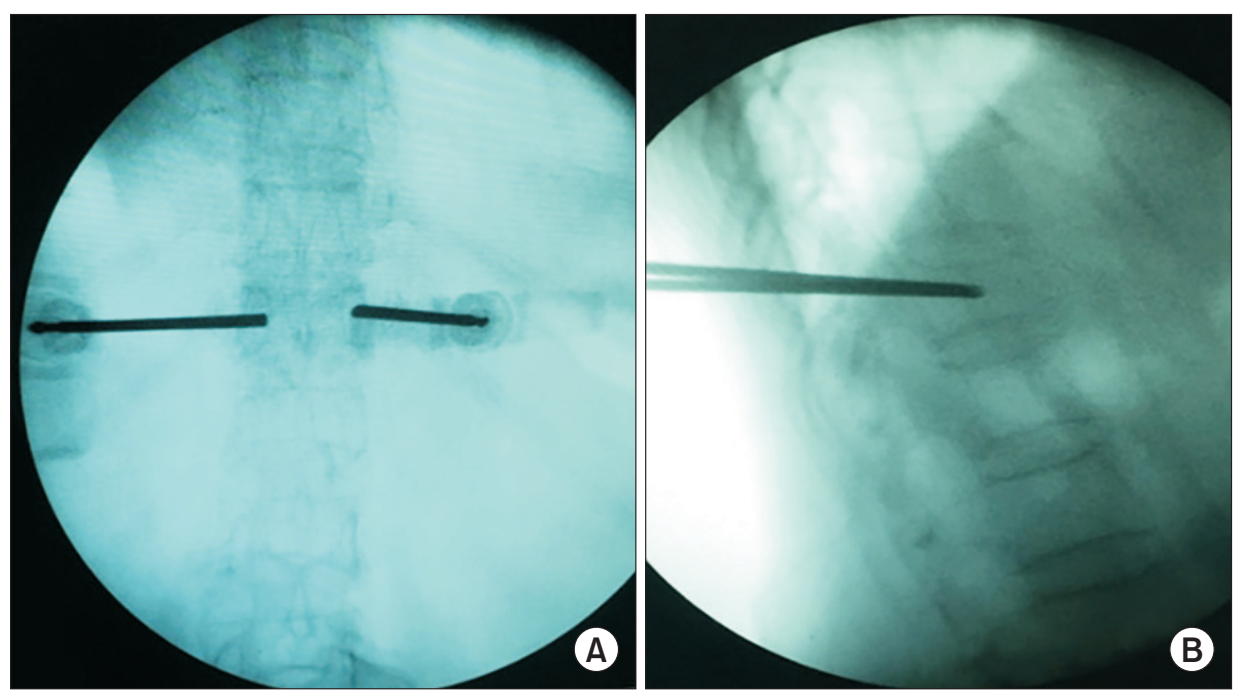

Fig. 1. The trocar was inserted through the skin to the pedicle. The trocar was tapped through the pedicle into the collapsed vertebral body. (A) The trocar was inserted through the skin to the pedicle. (B) The trocar was tapped through the pedicle into the collapsed vertebral body. 
Nguyen et al. Balloon Kyphoplasty in the Treatment of Osteoporotic Vertebral Compression Fractures

Clinics in Orthopedic Surgery • Vol. 12, No. 2, $2020 \bullet$ www.ecios.org

car. The trocar was inserted into position of the upper and outer halves of the joint to avoid damaging nerve roots and blood vessels in the vertebral cavity. The puncture of the vertebrae under the guidance of computed tomography (CT) allowed avoidance of damage to soft tissues and blood vessels.

Inflation of the balloon to create a space in the vertebral body

An inflatable balloon was inserted through the trocar into the vertebra. The balloon was slowly inflated with contrast dye to help restore the vertebral height and form a new cavity under the fluoroscopic guidance. The bone cement was then injected into the newly created cavity and quickly hardened (Fig. 2).

\section{Injection of bone cement}

Mixed cement was slowly injected via the trocar and controlled continuously under fluoroscopic guidance in an inclined position. The deepest area was filled first, and then the needle was withdrawn slightly to fill the upper areas. If the cement spills into a vein around the vertebra, we stopped to injection for a few seconds and then continued (Fig. 3).

\section{Clinical Outcome Evaluation}

Imaging follow-up consisted of anteroposterior and lateral spinal X-ray examinations at 24 hours and 3 months after the procedure. MRI was also performed before the procedure and at 24 hours and 3 months after the procedure in all patients. The patient's pain level was assessed with a visual analog scale (VAS) before surgery and at 24 hours and 3 months after the procedure. The effectiveness of the treatment in the study population was assessed according to Macnab's classification. ${ }^{10)}$

\section{Statistical Analysis}

Descriptive data were presented as means and standard
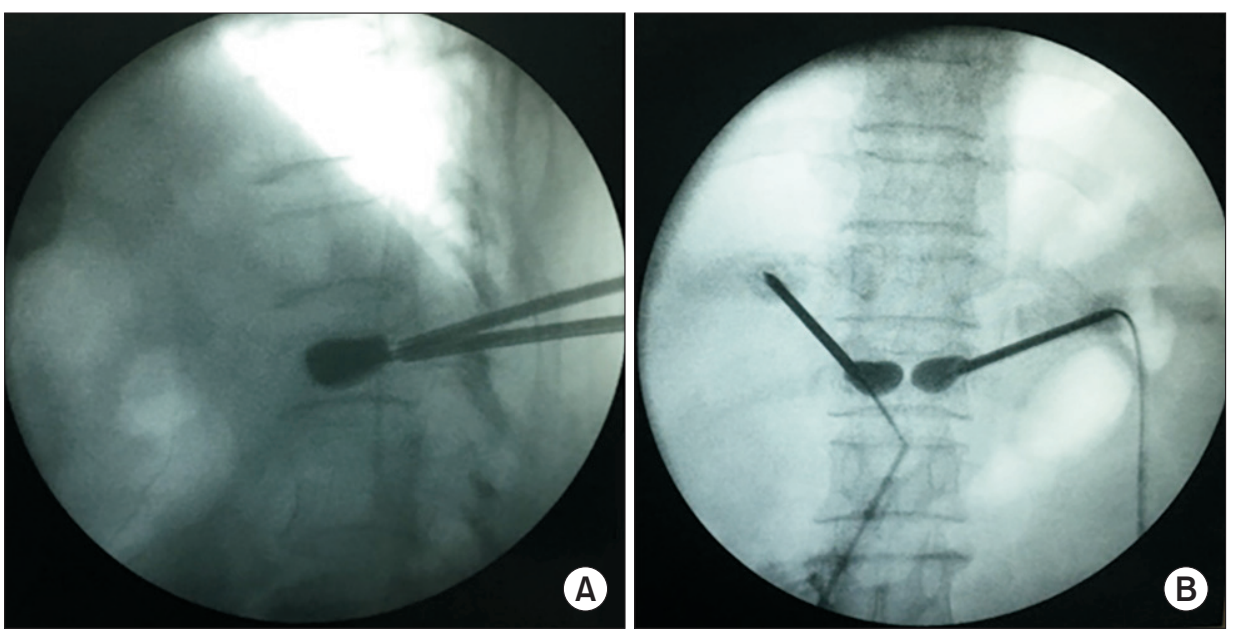

Fig. 2. The balloon was inflated to help restore vertebral height and form a new cavity. Lateral image (A) and anteroposterior image (B) after inflation of the balloon.
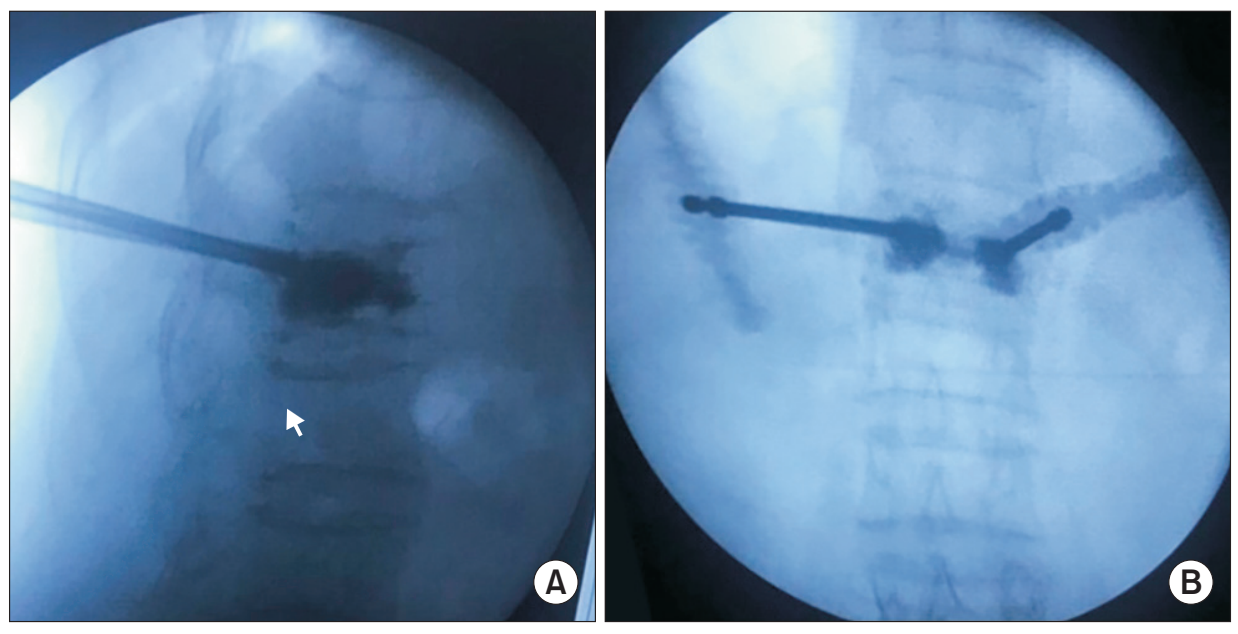

Fig. 3. Bone cement injected into the cavity. Lateral image (A) and anteroposterior image (B) after injection of bone cement. Arrow: upper edge of the lower vertebral body. 
Nguyen et al. Balloon Kyphoplasty in the Treatment of Osteoporotic Vertebral Compression Fractures

Clinics in Orthopedic Surgery • Vol. 12, No. 2, $2020 \bullet$ www.ecios.org

deviations (SDs) or percentages. All data were analyzed with Stata ver. 15 (Stata Corp., College Station, TX, USA). A $p$-value of $<0.05$ was considered statistically significant.

\section{Ethics}

All clinical investigations were conducted according to the principles expressed in the Declaration of Helsinki. Hanoi Medical University Institutional Review Board Committee approved the study protocol (IRB No. 4383/ QD-DHYHN). Informed consents were obtained from all individual participants included in the study.

\section{RESULTS}

\section{Clinical and Subclinical Characteristics}

From June 2018 and May 2019, a total of 65 patients were enrolled and underwent treatment with BKP. Their mean age was 69.9 years (SD, 10.7 years; range, $49-92$ years).

Table 1. Clinical Characteristics of Patients $(n=65)$

\begin{tabular}{|c|c|}
\hline Characteristic & No. $(\%)$ \\
\hline Age (yr), mean $\pm S D$ (range) & $69.9 \pm 10.7(49-92)$ \\
\hline Female sex & 58 (89.2) \\
\hline \multicolumn{2}{|l|}{ Past medical history } \\
\hline At least one medical treatment & $29(44.6)$ \\
\hline Previous VCF & $16(24.6)$ \\
\hline Previous kyphoplasty & $6(9.2)$ \\
\hline Onset of VCF after trauma & $47(72.3)$ \\
\hline \multicolumn{2}{|l|}{ Clinical symptom } \\
\hline Back pain & $65(100)$ \\
\hline Limited spinal mobility & $46(70.8)$ \\
\hline Chest pain $\&$ shortness of breath & $2(3.1)$ \\
\hline Visual analog scale grading ${ }^{*}$, mean \pm SD & $7.3 \pm 1.1$ \\
\hline 0 & 0 \\
\hline $1-2$ & 0 \\
\hline $3-4$ & 0 \\
\hline $5-6$ & $19(29.2)$ \\
\hline $7-8$ & $39(60.0)$ \\
\hline $9-10$ & $7(10.8)$ \\
\hline Pain duration (day), mean $\pm S D$ (interquartile range) & $14.3 \pm 20.2(1-90)$ \\
\hline
\end{tabular}

SD: standard deviation, VCF: vertebral compression fracture.

*Based on the number of points scored.
Most patients were women (89.2\%). On the past medical history reported by patients, $44.6 \%$ of the patients experienced at least one medical treatment, $16.0 \%$ had previous VCF, and $6.0 \%$ had previous kyphoplasty. There were 47 patients reporting the onset of VCF after trauma (72.3\%). Clinical symptoms at baseline were back pain (100\%), limited spinal mobility (70.8\%), and chest pain and shortness of breath (3.1\%). The mean VAS score was $7.3 \pm 1.1$. The mean duration of pain was 14.3 days (SD, 20.2 days; range, 1-90 days) (Table 1 ).

Subclinical characteristics are shown in Table 2. In the analysis of T-score, six patients who underwent previous kyphoplasty treatment were excluded because their T-scores might be influenced by previous bone cement injection. The mean T-score of the remaining 59 patients was -3.6 (SD, 0.8 ; range, -2.5 to -6.8 ). Most patients had

\section{Table 2. Subclinical Characteristics of Patients}

\begin{tabular}{|lc|}
\hline \multicolumn{1}{|c}{ Characteristic } & No. $(\%)$ \\
\hline Patient $(\mathrm{n}=65)$ & \\
\hline Bone density T-score, mean $\pm S D$ (range) & $-3.6 \pm 0.8(-2.5$ to -6.8$)$ \\
\hline Number of compressed thoracic vertebrae & \\
\hline Vertebra & $5(7.7)$ \\
\hline 2 Vertebrae & $2(3.1)$ \\
\hline 3 Vertebrae & $16.2 \pm 8.4(2.3$ to 43.3$)$ \\
\hline Cobb angle $\left(^{\circ}\right)$, mean $\pm S D$ (range) & \\
\hline Compressed thoracic vertebra $(n=73)$ & \\
\hline Thoracic vertebral level & $1(1.4)$ \\
\hline T5 & $1(1.4)$ \\
\hline T6 & $4(5.5)$ \\
\hline T7 & $4(5.5)$ \\
\hline T8 & $6(8.2)$ \\
\hline T9 & $3(4.1)$ \\
\hline T10 & $11(15.0)$ \\
\hline T11 & $43(58.9)$ \\
\hline T12 & \\
\hline Grade of fracture & $41(56.2)$ \\
\hline Grade 1 & $26(35.6)$ \\
\hline Grade 2 & $6(8.2)$ \\
\hline Grade 3 & \\
\hline
\end{tabular}

SD: standard deviation. 
Nguyen et al. Balloon Kyphoplasty in the Treatment of Osteoporotic Vertebral Compression Fractures

Clinics in Orthopedic Surgery • Vol. 12, No. 2, $2020 \bullet$ www.ecios.org

one compressed vertebra (89.2\%), five patients had two compressed vertebrae (7.7\%), and two patients had three compressed vertebrae (3.1\%). The mean Cobb angle was $16.2^{\circ}\left(\mathrm{SD}, 8.4^{\circ}\right.$; range, $\left.2.3^{\circ}-43.3^{\circ}\right)$. In the 73 thoracic vertebrae, bone cement was injected mostly at T11 and T12 levels (73.9\%); in $12.3 \%$, it was injected at T9 and T10 levels and in $13.8 \%$, at T5-T8 levels. None of the compression was observed above the T4 level. According to the Kannis's classification of VCF, $56.2 \%$ of the compressed vertebrae was grade $1,35.6 \%$ was grade 2 , and $8.2 \%$ was grade 3 .

\section{Technique of Restoring the Vertebral Height with BKP}

The trocar was inserted in $84.9 \%$ of the collapsed vertebrae through the pedicle and in $15.1 \%$, beside the pedicle. The mean amount of injected cement was $4.1 \mathrm{~mL}$ (SD, 1.1 $\mathrm{mL}$; range, $1.5-7 \mathrm{~mL}$ ). Forty vertebrae had cement infiltration over $2 / 3$ (54.8\%), 32 vertebrae had cement infiltration between $1 / 3$ and $2 / 3(43.8 \%)$, and only one vertebra had cement infiltration less than $1 / 3(1 / 4 \%)$ (Table 3$)$.

\section{Treatment Outcomes}

Compared with the baseline score, improvement in VAS after BKP was not statistically significant within 24 hours $(3.3 \pm 0.6, p<0.001)$ and at 3 months $(1.2 \pm 1.1, p<0.001)$. The treatment effect evaluated according to Macnab's classification was excellent in $40 \%$ of patients, well in $49.2 \%$, and fair in $10.8 \%$; there was no case with poor treatment effect. Two types of complications that occurred after BKP were intercostal neuralgia (3.1\%) and cement leakage (30.8\%) (Table 4).

Table 3. Technique for Restoring the Vertebral Height with Balloon Kyphoplasty $(n=73)$

\begin{tabular}{|cc|}
\hline \multicolumn{1}{|c}{ Variable } & No. (\%) \\
\hline Trocar insertion position into the vertebra & \\
\hline Trocar insertion through pedicle & $11(15.1)$ \\
\hline Trocar insertion beside pedicle & \\
\hline Amount of cement injected (mL), mean \pm SD (range) & $4.1 \pm 1.1(1.5-7)$ \\
\hline Infiltration of cement in vertebral body & $1(1.4)$ \\
\hline Less than $1 / 3$ & $32(43.8)$ \\
\hline From $1 / 3$ to $2 / 3$ & $40(54.8)$ \\
\hline Over $2 / 3$ &
\end{tabular}

SD: standard deviation.

\section{DISCUSSION}

Of the 65 patients, there were 47 patients (72.3\%) reporting the onset of VCF after trauma. Similar findings were reported by Weycker et al. ${ }^{11)}$ and Do. ${ }^{12)}$ Such high figures might be explained by the fact that Viet Duc Hospital is the last level surgical facility in Vietnamese health system; as a result, the patients with spinal injuries were referred more often to the hospital. After enrolling all patients with osteoporosis, we found that the osteoporotic thoracic VCFs recorded happened after minor injuries. Most patients in the study had one vertebra compressed (89.2\%), while a small number of patients had two to three vertebrae compressed. This result is consistent with previous findings. ${ }^{12,13)}$ The presence of edema on MRI indicates the novelty of the injury that causes back pain. In these newly compressed vertebrae, significant improvement, such as pain relief, can be expected after treatment. Compressed vertebrae without bone marrow edema on MRI often reflect chronic compression of the vertebrae. In such chronic cases, the bone in the vertebrae is healed very solid; since the trocar cannot be inserted into the vertebral body, these cases are not indicated for vertebral augmentation. As shown in our study, the Vietnamese patients with VCFs often suffer from pain long before visiting the hospital. It is worth noting that the mean duration of pain in our patients was $14.3 \pm 20.2$ days. This was because either patients self-treated at home or underwent treatment at a medical facility that was not capable of surgery. Only when those treatments failed, did the patients move to a higher

\section{Table 4. Treatment Outcomes $(n=65)$}

\begin{tabular}{lc}
\multicolumn{1}{c}{ Outcome } & Value \\
Visual analog scale score & \\
Within 24 hours & $3.3 \pm 0.6$ \\
3 Months & $1.2 \pm 1.1$ \\
\hline Macnab criteria & \\
\hline I (excellent) & $26(40.0)$ \\
\hline II (good) & $32(49.2)$ \\
\hline III (fair) & $7(10.8)$ \\
\hline IV (poor) & 0 \\
\hline Complication & \\
\hline Intercostal neuralgia & $2(3.1)$ \\
\hline Cement leakage & $20(30.8)$ \\
\hline
\end{tabular}

Values are presented as mean \pm standard deviation or number (\%). 
Nguyen et al. Balloon Kyphoplasty in the Treatment of Osteoporotic Vertebral Compression Fractures

Clinics in Orthopedic Surgery • Vol. 12, No. 2, $2020 \bullet$ www.ecios.org

level of health care facility for BKP.

There were 65 consecutive patients and the number of collapsed vertebrae with cement injection was 73. Under the fluoroscopic guidance, the trocar was inserted in $84.9 \%$ of the collapsed vertebrae (62/73) through the pedicle and in $15.1 \%$ (11/73), beside the pedicle. According to Boszczyk et al., ${ }^{14)}$ the mid-thoracic vertebrae (T5-T8) and the high segmental thoracic vertebrae (T1-T4) have slender stalks, which restrict access by poking the trocar through the pedicle, requiring an external trocar poke. In our study, one patient with a small T9 stalk, and other patients with high and medium thoracic vertebrae (T5T8) were subjected to a pedial trocar puncture to enter the vertebrae. Meanwhile, in the remaining patients with vertebral collapse from T9-T12 with large stalks, bone cement was injected through the trocar through the stem. For 73 compressed vertebrae, the mean amount of mixed cement injected was $4.1 \pm 1.1 \mathrm{~mL}$. To assess the extent of cement infiltration in the thoracic vertebral body, we used spinal X-ray after the cement injection, which showed 40 vertebrae with cement infiltration over $2 / 3$ (54.8\%), 32 vertebrae with cement infiltration between $1 / 3$ and $2 / 3$ (43.8\%), and only one vertebra had cement infiltration less than $1 / 3(1 / 4 \%)$. In a study by Ge et al. ${ }^{15)}$ on 38 patients with 52 collapsed thoracic vertebrae shaped by BKP, the mean amount of cement injected into the vertebral body was $3.2 \pm 1.4 \mathrm{~mL}$. In our study, we did not find a significant correlation between the amount of bone cement injected and the cement infiltration rate in the vertebrae. It might be because the amount of cement injected into the vertebral body depended on the degree of fracture, the lesions on the diagnostic imaging, and the surgeon's experience. Because of the sharp decrease in bone density in the study population diagnosed with osteoporotic thoracic VCFs, usually much amount of cement was injected into the vertebral body.

Even though kyphoplasty has significantly lower rates of cement extravasation than vertebroplasty, ${ }^{16)}$ cement leakage may occur more frequently and it may be associated with a significant morbidity. ${ }^{17)}$ In addition, systemic allergic or toxic reactions to polymethyl methacrylate monomers have been described. ${ }^{18)}$ Hence, the common feared complication of all vertebral augmentation techniques is polymethyl methacrylate monomers cement leakage. A systematic review by Hulme et al. ${ }^{17)}$ reported the rate of cement leakage in vertebroplasty as $41 \%(\mathrm{n}=2,283$ levels $)$ and in kyphoplasty as 9\% ( $\mathrm{n}=$ 1,486 levels) of treated vertebrae. In the present study, radiographically confirmed cement leakage was in 30.8\% of all cases. Complications caused by cement extravasa- tion through the anterior margin of vertebrae accounted for $15.4 \%$; those through the vertebral disc, $12.3 \%$; and those through the posterior margin of vertebra, $3.1 \%$. This indicates that cement extravasation through the anterior margin of vertebrae and vertebral disc is common. This might be explained by the high proportions of grade 1 compressed vertebrae (wedge-shaped collapse) and grade 2 compressed vertebrae (double-sided concave collapse) according to the Kannis's classification. Also, in the 3.1\% of patients who had cement extravasation in the posterior margin of vertebra, there was no clinically notable lesion of the nerve roots or spinal cord. In the patients with spinal injury with fracture lines or severe VCFs, the risk of posterior cement spill was increased. We attempted to limit posterior cement spill in these cases by injecting a small amount of cement slowly via the trocar; the injection of cement mix was controlled continuously under fluoroscopic guidance with the patient in an inclined position.

Pain intensity was assessed by VAS in all patients at 24 hours and 3 months after BKP. We found a statistically significant reduction in the mean VAS from $7.3 \pm 1.1$ preoperatively to $3.3 \pm 0.6$ at 24 hours and then to $1.2 \pm 1.1$ at 3 months after procedure $(p<0.01)$. In a study of Robinson et al. ${ }^{19)}$ on 102 VCF patients undergoing BKP treatment, the preoperative VAS was $7.5 \pm 1.3$. Postoperative pain levels were significantly reduced 1 day after surgery (VAS, $2.3 \pm 2.2$ ) and at the 6-month follow-up (VAS, 1.4 $\pm 0.9) .{ }^{19)}$ In Baz et al.' study ${ }^{20)}$ on 82 patients with VCF, the mean VAS was 7.97, which decreased to 2.21 after a surgical operation and then to 1.98 at the follow-up period. Our results are consistent with those of previous studies, indicating that the VAS rapidly decreased after the injection of cement. To evaluate the efficiency of restoring the height of the vertebral body with BKP, we used the Macnab's classification. We found excellent treatment results in $40 \%$, good results in $49.2 \%$, and fair results in $10.8 \%$. Most patients achieved good and excellent treatment results, while those with fair treatment results were diagnosed with severe VCFs, were in weak physical condition, and had at least a comorbid condition. We found a reduction of $3.7^{\circ}$ in Cobb angle assessed on standing radiographs taken after BKP $(p<0.01)$. Similar results were reported in previous studies: a decrease of $2^{\circ}$ in Boszczyk et al.s, ${ }^{14)} 8^{\circ}$ in Xiong et al.s, ${ }^{21)}$ and $3.1^{\circ}$ in Voggenreiter's. ${ }^{22)}$ These findings indicate that the treatment using BKP for thoracic VCFs due to osteoporosis is effective in improving the Cobb angle of the injury area.

To the best of our knowledge, this is the first study that focuses on Vietnamese patients who were diagnosed with osteoporotic thoracic VCFs and treated with BKP. 
Nguyen et al. Balloon Kyphoplasty in the Treatment of Osteoporotic Vertebral Compression Fractures

Clinics in Orthopedic Surgery • Vol. 12, No. 2, $2020 \bullet$ www.ecios.org

The important limitations of the study that need to be clearly acknowledged include that the number of patients was relatively small and no control group was included. In future randomized multicenter trials on BKP in Vietnamese patients, a comparison with a more representative sample size is required to evaluate the mid- and long-term clinical efficacy, as well as the risk of complications associated with the treatment of osteoporotic thoracic VCFs.

In conclusions, the evidence for the efficacy and safety of BKP in Vietnamese patients is sparse. Our study results support the fact that height restoration of thoracic osteoporotic VCF with BKP is a minimally invasive treatment that is effective for immediate pain relief, early motor rehabilitation, and humpback correction. The present study provided convincing evidence to support the use of BKP by spine surgeons and clinical specialists in treat- ing osteoporotic thoracic VCFs in Vietnamese patients. Also, our findings could serve as a cornerstone to promote further studies and to motivate Vietnamese physicians to apply evidence-based clinical practice among a variety of conservative treatment options.

\section{CONFLICT OF INTEREST}

No potential conflict of interest relevant to this article was reported.

\section{ACKNOWLEDGEMENTS}

We thank all patients enrolled in this study and their families for their support of the research.

\section{REFERENCES}

1. Varacallo MA, Fox EJ. Osteoporosis and its complications. Med Clin North Am. 2014;98(4):817-31.

2. Silverman SL. The clinical consequences of vertebral compression fracture. Bone. 1992;13 Suppl 2:S27-31.

3. Marcucci G, Brandi ML. Kyphoplasty and vertebroplasty in the management of osteoporosis with subsequent vertebral compression fractures. Clin Cases Miner Bone Metab. 2010;7(1):51-60.

4. Eck JC, Nachtigall D, Humphreys SC, Hodges SD. Comparison of vertebroplasty and balloon kyphoplasty for treatment of vertebral compression fractures: a meta-analysis of the literature. Spine J. 2008;8(3):488-97.

5. Lavelle WF, Khaleel MA, Cheney R, Demers E, Carl AL. Effect of kyphoplasty on survival after vertebral compression fractures. Spine J. 2008;8(5):763-9.

6. Galibert P, Deramond H, Rosat P, Le Gars D. Preliminary note on the treatment of vertebral angioma by percutaneous acrylic vertebroplasty. Neurochirurgie. 1987;33(2):166-8.

7. Kasper DM. Kyphoplasty. Semin Intervent Radiol. 2010;27(2):172-84.

8. Edidin AA, Ong KL, Lau E, Kurtz SM. Mortality risk for operated and nonoperated vertebral fracture patients in the medicare population. J Bone Miner Res. 2011;26(7):161726.

9. Medical Advisory Secretariat. Balloon kyphoplasty: an evidence-based analysis. Ont Health Technol Assess Ser. 2004;4(12):1-45.

10. Macnab I. Chapter 14. Pain and disability in degenerative disc disease. Clin Neurosurg. 1973;20:193-6.

11. Weycker D, Li X, Barron R, Bornheimer R, Chandler D. Hospitalizations for osteoporosis-related fractures: Economic costs and clinical outcomes. Bone Rep. 2016;5:18691.

12. Do MH. Clinical and subclinical characteristics and evaluation of results of the application of kyphoplasty in osteoporotic patients with vertebral compression fractures [dissertation]. Hanoi: Hanoi Medical University; 2009.

13. Ge Z, Ma R, Chen Z, et al. Uniextrapedicular kyphoplasty for the treatment of thoracic osteoporotic vertebral fractures. Orthopedics. 2013;36(8):e1020-4.

14. Boszczyk BM, Bierschneider M, Hauck S, Beisse R, Potulski M, Jaksche H. Transcostovertebral kyphoplasty of the mid and high thoracic spine. Eur Spine J. 2005;14(10):992-9.

15. Ge J, Cheng X, Li P, Yang H, Zou J. The clinical effect of kyphoplasty using the extrapedicular approach in the treatment of thoracic osteoporotic vertebral compression fracture. World Neurosurg. 2019;131:e284-9.

16. Phillips FM, Todd Wetzel F, Lieberman I, Campbell-Hupp M. An in vivo comparison of the potential for extravertebral cement leak after vertebroplasty and kyphoplasty. Spine (Phila Pa 1976). 2002;27(19):2173-9.

17. Hulme PA, Krebs J, Ferguson SJ, Berlemann U. Vertebroplasty and kyphoplasty: a systematic review of 69 clinical studies. Spine (Phila Pa 1976). 2006;31(17):1983-2001.

18. Kalteis T, Luring C, Gugler G, et al. Acute tissue toxicity of PMMA bone cements. Z Orthop Ihre Grenzgeb. 2004;142(6):666-72. 
Nguyen et al. Balloon Kyphoplasty in the Treatment of Osteoporotic Vertebral Compression Fractures

Clinics in Orthopedic Surgery • Vol. 12, No. 2, $2020 \bullet$ www.ecios.org

19. Robinson Y, Tschoke SK, Stahel PF, Kayser R, Heyde CE. Complications and safety aspects of kyphoplasty for osteoporotic vertebral fractures: a prospective follow-up study in 102 consecutive patients. Patient Saf Surg. 2008;2:2.

20. Baz AB, Akalin S, Kilicaslan OF, Tokatman B, Arik H, Duygun F. Efficiency of balloon kyphoplasty in the treatment of osteoporotic vertebral compression fractures. Kobe J Med Sci. 2016;62(3):E49-54.
21. Xiong J, Dang Y, Jiang BG, Fu ZG, Zhang DY. Treatment of osteoporotic compression fracture of thoracic/lumbar vertebrae by kyphoplasty with SKY bone expander system. Chin J Traumatol. 2010;13(5):270-4.

22. Voggenreiter G. Balloon kyphoplasty is effective in deformity correction of osteoporotic vertebral compression fractures. Spine (Phila Pa 1976). 2005;30(24):2806-12. 